\title{
Religiosidade e/ou espiritualidade como fatores de proteção ao uso de álcool entre adolescentes: revisão sistemática
}

\author{
Religiosity and/or spirituality as protection factors for the use of alcohol among adolescents: systematic review \\ Religiosidad y / o espiritualidad como factores de protección del uso de alcohol en adolescentes: revisión sistemática
}

\begin{abstract}
RESUMO
Objetivo: identificar evidências da influência da religiosidade e/ou espiritualidade como fatores de proteção ao uso de álcool em adolescentes. Método: Revisão Sistemática da Literatura. Bases elegíveis: PubMed, Lilacs, Cinahl e Embase. Cadastrou-se, esta revisão no banco de dados PROSPERO, que recebeu protocolo n CRD42020188122, e seguiu a adequação do protocolo PRISMA. A busca inicial resultou em 1.180 artigos. Após a leitura dos títulos e resumos, restaram 179 artigos. Após aplicação dos critérios de inclusão e exclusão, foram selecionados 09 estudos finais. Resultados: Identificou-se, a religiosidade e espiritualidade como um importante fator de proteção aos adolescentes contra o uso do álcool. Conclusão: como sugerem os resultados deste estudo, a religiosidade e a espiritualidade configuraram-se como fatores de proteção ao uso do álcool por adolescentes. Nesse sentido, se pesquisas adicionais apoiarem essa hipótese, a identificação de tais adolescentes será importante para destinar prioritariamente os esforços de prevenção. Não obstante, o grande desafio é traduzir esses achados em programas eficazes de prevenção.

DESCRITORES: Espiritualidade; Religião; Adolescentes; Álcool.
\end{abstract}

\section{ABSTRACT}

Objective: identify evidence of the influence of religiosity and/or spirituality as protective factors against alcohol use in adolescents. Methods: Systematic Literature Review. Elegible bases: PubMed, Lilacs, Cinahl and Embase databases. This review was registered in the PROSPERO database, which received protocol number CRD42020188122, and followed the adequacy of the PRISMA protocol. The initial search resulted in 1,180 articles. After reading the titles and abstracts, 179 articles remained. After applying the inclusion and exclusion criteria, 09 final studies were selected. Results: Religiosity and spirituality were identified as an important protective factor for adolescents against alcohol use. Conclusion: as the results of this study suggest, religiosity and spirituality were configured as protective factors against alcohol use by adolescents. In this sense, if additional research supports this hypothesis, the identification of such adolescents will be important to prioritize prevention efforts. However, the great challenge is to translate these findings into effective prevention programs.

DESCRIPTORS: Spirituality; Religion; Adolescent; Alcohol.

\section{RESUMEN}

Objetivo: identificar evidencias de la influencia de la religiosidad y / o espiritualidad como factores protectores contra el consumo de alcohol en adolescentes. Métodos: Revisión sistemática de la literatura. Bases elegibles: PubMed, Lilacs, Cinahl y Embase. Esta revisión se registró en la base de datos PROSPERO, que recibió el número de protocolo CRD42020188122, y siguió la adecuación del protocolo PRISMA. La búsqueda inicial dio como resultado 1.180 artículos. Después de leer los títulos y resúmenes, quedaron 179 artículos. Después de aplicar los criterios de inclusión y exclusión, se seleccionaron 09 estudios finales. Resultados: La religiosidad y la espiritualidad se identificaron como un factor protector importante para los adolescentes contra el consumo de alcohol. Conclusión: como sugieren los resultados de este estudio, la religiosidad y la espiritualidad se configuraron como factores protectores frente al consumo de alcohol por parte de los adolescentes. En este sentido, si la investigación adicional apoya esta hipótesis, la identificación deT estos adolescentes será importante para priorizar los esfuerzos de prevención. Sin embargo, el gran desafío es traducir estos hallazgos en programas de prevención efectivos.

DESCRIPTORES: Espiritualidad; Religión; Adolescentes; Alcohol.

RECEBIMENTO: 18/09/2020 APROVAÇÃO: 30/09/2020

\section{Lázaro Clarindo Celestino}

Enfermeiro. Mestre em Enfermagem pela Escola de Enfermagem de Ribeirão Preto da Universidade de São Paulo, EERP-USP. Doutorando em Ciências da Reabilitação pelo Hospital de Reabilitação de Anomalias Craniofaciais da Universidade de São 
Paulo, HRAC-USP.

ORCID: 0000-0002-4900-8164.

\section{Ana Paula Fukushiro}

Fonoaudióloga. Professora Assistente. Departamento de Fonoaudiologia da Faculdade de Odontologia de Bauru, Universidade de São Paulo. Programa de Pós-Graduação em Ciências da Reabilitação, Hospital de Reabilitação de Anomalias Craniofaciais, Universidade de São Paulo, São Paulo (SP) Brasil.

ORCID: 0000-0002-5927-3877.

\section{Andressa Sharllene Carneiro da Silva}

Fonoaudióloga. Doutoranda em Ciências da Reabilitação pelo Hospital de Reabilitação de Anomalias Craniofaciais da Universidade de São Paulo, HRAC-USP. São Paulo, Brazil. ORCID:

0000-0003-1392-5478

\section{Joel Ferreira Santiago Júnior}

Cirurgião Dentista. Professor do Centro Universitário Sagrado Coração, São Paulo, Brasil.

ORCID: 0000-0003-1735-2224

\section{Armando dos Santos Trettene}

Enfermeiro. Programa de Pós-Graduação em Ciências da Reabilitação do Hospital de Reabilitação de Anomalias Craniofaciais da Universidade de São Paulo, HRAC-USP. São Paulo, Brazil.

ORCID: 0000-0002-9772-857X

\section{INTRODUÇÃO}

0 álcool é a substância psicoativa mais utilizada no mundo, sendo reconhecida universalmente como grave problema de saúde públical. Capaz de levar a dependência, pode acarretar problemas de ordem física, mental e social, além de ser responsável pela morte de milhões de pessoas 1,2.

Entre os desarranjos ocasionados por sua utilização incluem-se alterações do humor, do aparelho locomotor e prejuízo das habilidades mentais. Além disso, contribui para uma série de doenças crônicas, infecciosas e imunológicas 3,4 .

A utilização do álcool como principal droga lícita entre os adolescentes está consolidada na literatura. Sabe-se que sua experimentação tem ocorrido precocemente, geralmente entre 10 e 13 anos de idade, com predomínio aos 15 anos, apontando a gravidade desta problemática de abrangência mundial5-8.

O consumo precoce de álcool resulta em adoção de comportamentos de risco, incluindo o uso de drogas lícitas e ilícitas, prejuízos no desempenho escolar, gravidez precoce, acidentes de trânsito e violência de diferentes tipos9. Em associação, utilizar álcool na adolescência predispõe a piores níveis de saúde na vida adulta, além do uso abusivo10,11.

Embora se reconheçam progressos no tratamento dos problemas relacionados ao álcool, a prevenção continua sendo um desafio e o uso cada vez mais frequente entre adolescentes 12 .

Frente a essa realidade, diversos pesquisadores têm reunido esforços no sentido de identificar estratégias que possam prevenir ou minimizar o uso e/ou o abuso do álcool entre adolescentes. Nesse, uma série de pesquisas reforçam a hipótese de que espiritualidade e/ou a religiosidade atuam como fatores de proteção contra hábitos de vida deletérios5,13-17, incluindo o uso de álcool18-22.

A espiritualidade se refere "à busca pessoal para entender as questões sobre a vida, sobre seu sentido, sobre as relações com o sagrado ou transcendente, que pode ou não levar ao desenvolvimento de práticas religiosas". A religiosidade, no que lhe concerne, pode ser definida como a extensão na qual um indivíduo acredita, segue e pratica uma religião, podendo ser organizacional caracterizando-se pela participação ativa na igreja ou em templo religioso, ou não organizacional, manifestada por meio dos atos de rezar, ler livros sobre a temática, assistir a programas religiosos, entre outros 23 .
Em suma, a religiosidade caracteriza-se pela interação do indivíduo com grupos, enquanto a espiritualidade vincula-se a ações individuais. Devido ao fato da espiritualidade e da religiosidade compreenderem conceitos multifacetados e, por vezes, subjetivos, seus benefícios não estão completamente esclarecidos, assim como as variáveis correlatas, representando um campo a ser explorado24,25.

Sabe-se, por exemplo, que há diferenças dos níveis de proteção ao uso do álcool entre designações religiosas, fatores sociodemográficos e psicossociais, demonstrando a complexidade dessa temática e os desafios para sua elucidação 17,26.

Considerando-se os efeitos deletérios do álcool nos contextos biológico e psicossocial, assim como sua utilização crescente entre adolescentes em um contexto mundial, torna-se indispensável identificar possíveis fatores de proteção.

Assim, espera-se com este estudo, apresentar evidências sobre a influência da religiosidade e/ou da espiritualidade como fator de proteção para o uso do álcool entre adolescentes, cujos resultados poderão subsidiar políticas públicas e intervenções voltadas a esse público.

Este estudo teve o objetivo de identificar as evidências da influência da religiosidade 
e/ou da espiritualidade como fatores de proteção ao uso do álcool entre adolescentes.

\section{MÉTODO}

Trata-se de uma revisão sistemática da literatura, delineada segundo os critérios estabelecidos pela colaboração Cochrane Handbook for Systematic Reviews of Interventions - Handbook 5.1.0 (Cochrane)27 e das diretrizes estabelecidas pelo PRISMA, sendo para a metodologia o PRISMA-P e para resumo o PRISMA-A28-30.

Esta revisão sistemática foi cadastrada na International Prospective Register of Systematic Reviews - PROSPERO, sob o protocolo: CRD42020188122.

Para construção da pergunta de pesquisa utilizou-se a estratégia PICO, onde a população $(\mathrm{P})$ incluiu adolescentes com idades entre 10 a 19 anos, a intervenção (I) referiu-se a religiosidade e/ou a espiritualidade, a comparação $(\mathrm{C})$ foi entre adolescentes praticantes e não praticantes da religiosidade e/ou espiritualidade, enquanto o desfecho (O - outcomes) voltou-se a identificação dos efeitos da prática da religiosidade e/ou da espiritualidade no uso de álcool entre adolescentes. Em suma, buscou-se responder ao seguinte questionamento: a prática da religiosidade e/ou a espiritualidade atua como fator de proteção ao uso do álcool em adolescentes?

Foram incluídos estudos primários, nos idiomas português, espanhol e inglês, publicados de 31/01/2010 a 31/01/2020, cuja amostragem incluiu adolescentes entre 10 e 19 anos. Para estratificação da idade, considerou-se a padronização da Organização Mundial da Saúde. Foram excluídos estudos que não respondiam à pergunta da pesquisa e secundários, como os de revisão e opiniões de especialistas.

O levantamento dos dados foi realizado em 31 de janeiro de 2020, com auxílio de uma bibliotecária com vasta experiência. As bases de dados consultadas incluíram: Literatura Latino-Americana e do Caribe em Ciências da Saúde (LILACS), US National Library of Medicine (PubMed), Cumulative Index to Nursing and Allied Health

Quadro 1 - Estratégias de busca nas bases de dados. Bauru, SP, Brasil, 2020.

\begin{tabular}{|c|c|}
\hline PUBMED & $\begin{array}{c}\left(\left(\left(\left(\left(\text { ADOLESCEN }^{*} \mathrm{OR} \mathrm{TEEN}^{*} \mathrm{OR}_{\text {YOUTH}}^{*}\right)\right)\right)\right) \text { AND }\left(\left(\mathrm{SPIRITUAL}{ }^{*} \text { OR }\right.\right.\right. \\
\left.\left.\left.\text { RELIGI }^{*}\right)\right)\right) \text { AND ALCOHOL* }\end{array}$ \\
\hline Lilacs & $\begin{array}{l}\text { (tw:((Espiritual\$ OR Spiritual\$ OR Religi\$))) AND (tw:((adolescen\$ } \\
\text { OR teen\$ OR Youth\$))) AND (tw:((alcool\$ OR alcohol\$))) }\end{array}$ \\
\hline Cinahl & $\begin{array}{l}\text { ((adolescen* OR teen* OR Youth*)) AND ((Spiritual* OR Religi*)) AND } \\
\text { alcohol* }\end{array}$ \\
\hline Embase & $\begin{array}{l}\text { (adolescen* OR teen* OR youth*) AND (spiritual* OR religi*) AND } \\
\text { alcohol*. }\end{array}$ \\
\hline Fonte: Autoria. & \\
\hline
\end{tabular}

Quadro 2 - Níveis de evidência científica segundo a Classificação do Oxford Centre for Evidence Based Medicine segundo o tipo de estudo, Bauru, SP, Brasil, 2020.

\begin{tabular}{|c|c|c|}
\hline $\begin{array}{l}\text { GRAU DE } \\
\text { RECOMENDAÇÃO }\end{array}$ & $\begin{array}{l}\text { NIVEL DE } \\
\text { EVIDÊNCIA }\end{array}$ & TIPOS DE ESTUDOS \\
\hline \multirow{3}{*}{ A } & $1 A$ & $\begin{array}{l}\text { Revisão sistemática de ensaios clínicos controlados } \\
\text { e randomizados. }\end{array}$ \\
\hline & 1B & $\begin{array}{l}\text { Ensaio clínico controlado e randomizado com } \\
\text { intervalo de confiança estreito. Coorte validada, } \\
\text { com bom padrão de referência Critério Diagnóstico } \\
\text { testado em um único centro clínico. }\end{array}$ \\
\hline & $1 \mathrm{C}$ & Resultados terapêuticos do tipo "tudo ou nada". \\
\hline \multirow{5}{*}{ B } & $2 A$ & $\begin{array}{l}\text { Revisão Sistemática (com homogeneidade) de estu- } \\
\text { dos de coorte. }\end{array}$ \\
\hline & 2B & $\begin{array}{l}\text { Estudo de Coorte (incluindo Ensaio Clínico randomi- } \\
\text { zado de menor qualidade). }\end{array}$ \\
\hline & $2 \mathrm{C}$ & $\begin{array}{l}\text { Observação de resultados terapêuticos. Estudo Eco- } \\
\text { lógico. }\end{array}$ \\
\hline & $3 A$ & $\begin{array}{l}\text { Revisão Sistemática (com homogeneidade) de Estu- } \\
\text { dos Caso-Controle. }\end{array}$ \\
\hline & 3B & Estudo Caso-Controle. \\
\hline C & 4 & $\begin{array}{l}\text { Relato de Casos (incluindo coorte ou caso-controle } \\
\text { de menor qualidade) }\end{array}$ \\
\hline D & 5 & $\begin{array}{l}\text { Opinião desprovida de avaliação crítica ou baseada } \\
\text { em matérias básicas (estudo fisiológico ou estudo } \\
\text { com animais) }\end{array}$ \\
\hline
\end{tabular}

Literature (CINAHL) e Embase.

Os descritores foram selecionados por meio de consulta aos vocabulários Medical Subject Headings (MeSH) e Descritores em Ciências da Saúde (DeCS), sendo: espiritualidade, religião, adolescentes e álcool. Utilizou-se para a busca os descritores e seus sinônimos que foram combinados entre si com a utilização dos termos booleanos "AND" e "OR". As estratégias de busca encontram-se descritas no Quadro 1.

A extração e análise de dados foram realizadas por dois revisores, de forma independente, por meio de um formulário padronizado de coleta de dados que incluiu as seguintes variáveis: Título dos artigos, autores, país, ano das publicações, desenho dos estudos, amostra, idade dos participantes, grau de recomendação e nível de evidência, principais resultados e recomendações. Um 


\section{artigo}

Celestino, L. C.,Fukushiro, A. P., Silva, A. S. C., Júnior, J. F. S., Trettene, A. S.

Religiosidade e/ou espiritualidade como fatores de proteção ao uso de álcool entre adolescentes: revisão sistemática

terceiro pesquisador cruzou as informações coletadas para chegar a um consenso final sobre os dados extraídos.

Ressalta-se que não foi possível realizar a meta-análise devido a heterogeneidade clínica, variáveis e unidades de medidas distintas, assim como diferenças metodológicas.

O método utilizado para avaliar a qualidade do estudo, ou seja, do grau de recomendação e o nível de evidência foi o do Oxford Centre for Evidence Based Medicine, cuja metodologia encontra-se descrita no Quadro 231.

A revisão sistemática da literatura constitui estudo secundário, portanto, não foi necessária a avaliação do Comitê de Ética em Pesquisa, em conformidade aos preceitos da Resolução 466/2012 do CNS.

\section{RESULTADOS}

Inicialmente foram selecionados 1.180 artigos. Destes, após a leitura dos títulos e resumos selecionou-se 179 estudos. Por fim, 9 estudos compuseram a amostra final, conforme apresentado na Figura 1.

Dentre os nove artigos selecionados, o
Figura 1 - Diagrama PRISMA indicando a seleção dos artigos propostos. Bauru, SP, Brasil, 2021.

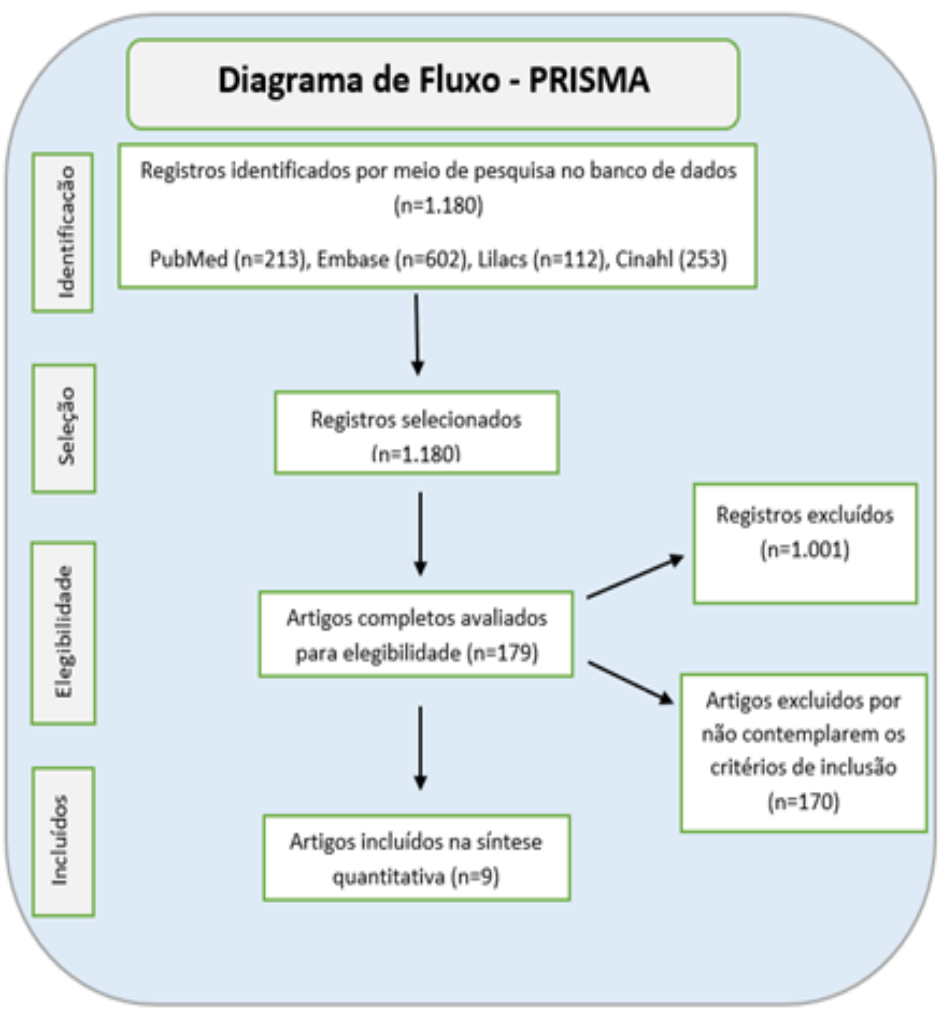

\section{Quadro 1 - Estratégias de busca nas bases de dados. Bauru, SP, Brasil, 2020.}

\begin{tabular}{|c|c|c|c|c|}
\hline $\begin{array}{l}\text { TÍTULO DO } \\
\text { ARTIGO }\end{array}$ & $\begin{array}{l}\text { AUTORES/ } \\
\text { PAÍS/ANO DA } \\
\text { PUBLICAÇÃOO }\end{array}$ & $\begin{array}{l}\text { DESENHO DO } \\
\text { ESTUDO/ AMOSTA/ } \\
\text { IDADE DOS } \\
\text { PARTICIPANTES }\end{array}$ & $\begin{array}{c}\text { GRAU DE } \\
\text { RECOMENDAÇÃO E } \\
\text { NÍVEL DE EVIDÊNCIA }\end{array}$ & $\begin{array}{l}\text { PRINCIPAIS RESULTADOS/ } \\
\text { RECOMENDAÇÕES }\end{array}$ \\
\hline $\begin{array}{l}\text { Religiosity, heavy } \\
\text { alcohol use, and } \\
\text { vicarious learning } \\
\text { networks among } \\
\text { adolescents in the } \\
\text { United States. }\end{array}$ & $\begin{array}{l}\text { Gryczynski J, Ward } \\
\text { BW25. EUA. } 2012 .\end{array}$ & $\begin{array}{l}\text { Estudo descritivo e } \\
\text { transversal. Amostra: } \\
14.556 \text { adolescentes. } \\
\text { Idade: } 12 \text { a } 17 \text { anos. }\end{array}$ & $B ; 2 C$ & $\begin{array}{l}\text { A religiosidade foi efetiva para pre- } \\
\text { venção do consumo de álcool em to- } \\
\text { dos os grupos analisados. }\end{array}$ \\
\hline $\begin{array}{l}\text { Does religiosity } \\
\text { delay adolescent } \\
\text { alcohol initiation? a } \\
\text { long-term analysis } \\
\text { (2008-2015) } \\
\text { of nationally } \\
\text { representatives } \\
\text { sample of 12th } \\
\text { graders. }\end{array}$ & $\begin{array}{l}\text { Barry AE, Valdez D, } \\
\text { Russel AM14. EUA. } \\
2020 .\end{array}$ & $\begin{array}{l}\text { Estudo descritivo } \\
\text { prospectivo. Amostra: } \\
2.374 \text { adolescentes. } \\
\text { Idade: } 17 \text { a } 18 \text { anos. }\end{array}$ & $B ; 2 C$ & $\begin{array}{l}\text { A espiritualidade se mostrou mais } \\
\text { efetiva na prevenção e proteção ao } \\
\text { uso do álcool quando comparada com } \\
\text { a religiosidade. }\end{array}$ \\
\hline
\end{tabular}




\begin{tabular}{|c|c|c|c|c|}
\hline $\begin{array}{l}\text { Alcohol consump- } \\
\text { tion among rural } \\
\text { African American } \\
\text { and white adoles- } \\
\text { cents: the role of } \\
\text { religion, parents, } \\
\text { and peers. }\end{array}$ & $\begin{array}{l}\text { Dickens DD, Jackman } \\
\text { DM, Stanley LR, Swa- } \\
\text { im RC, Chaves EL26. } \\
\text { EUA. 2018. }\end{array}$ & $\begin{array}{l}\text { Estudo descritivo trans- } \\
\text { versal. Amostra: } 23.163 \\
\text { adolescentes. Idade: } 12 \\
\text { a } 18 \text { anos. }\end{array}$ & $\mathrm{B} ; 2 \mathrm{C}$ & $\begin{array}{l}\text { A religiosidade foi efetiva para pre- } \\
\text { venção do uso de álcool para adoles- } \\
\text { centes afro-americanos e brancos de } \\
\text { comunidades rurais. }\end{array}$ \\
\hline $\begin{array}{l}\text { Religiosity, alcohol } \\
\text { use attitudes, and } \\
\text { alcohol use in a } \\
\text { national sample of } \\
\text { adolescents. }\end{array}$ & $\begin{array}{l}\text { Vaughan EL, Dios } \\
\text { MAD, Steinfeld JÁ, } \\
\text { Kratz LM32. EUA. } \\
\text { 2011. }\end{array}$ & $\begin{array}{l}\text { Estudo descritivo trans- } \\
\text { versal. Amostra: } 14.297 \\
\text { adolescentes. Idade: } 12 \\
\text { a } 17 \text { anos. }\end{array}$ & $\mathrm{B} ; 2 \mathrm{C}$ & $\begin{array}{l}\text { A religiosidade influênciou o uso e a } \\
\text { frequência de uso do álcool por ado- } \\
\text { lescentes. }\end{array}$ \\
\hline $\begin{array}{l}\text { To believe or not to } \\
\text { believe?" religio- } \\
\text { sity, spirituality, } \\
\text { and alcohol use } \\
\text { among Hungarian } \\
\text { adolescents. }\end{array}$ & $\begin{array}{l}\text { Piko BF, Kovacs E, } \\
\text { Kriston P, Fitzpatri- } \\
\text { ckKM33. Hungria. } \\
\text { 2012. }\end{array}$ & $\begin{array}{l}\text { Estudo descritivo trans- } \\
\text { versal. Amostra: } 592 \\
\text { adolescentes. Idade: } 14 \\
\text { a } 17 \text { anos. }\end{array}$ & $\mathrm{B} ; 2 \mathrm{C}$ & $\begin{array}{l}\text { A religiosidade e a espiritualidade } \\
\text { atuaram como fatores de proteção } \\
\text { dos adolescentes contra o uso de ál- } \\
\text { cool. }\end{array}$ \\
\hline $\begin{array}{l}\text { Are there gender, } \\
\text { racial, or religious } \\
\text { denominational } \\
\text { differences in } \\
\text { religiosity's effect } \\
\text { on alcohol use } \\
\text { and binge drinking } \\
\text { among youth in } \\
\text { the United States? } \\
\text { a propensity score } \\
\text { weighting appro- } \\
\text { ach. }\end{array}$ & Hai AH34. EUA. 2019. & $\begin{array}{l}\text { Estudo descritivo pros- } \\
\text { pectivo. Amostra: } 1.969 \\
\text { adolescentes. Idade: } 13 \\
\text { a } 17 \text { anos. }\end{array}$ & $\mathrm{B} ; 2 \mathrm{C}$ & $\begin{array}{l}\text { A religiosidade comportou-se como } \\
\text { fator de proteção ao uso de álcool por } \\
\text { adolescentes, a depender do sexo e } \\
\text { denominação religiosa. }\end{array}$ \\
\hline $\begin{array}{l}\text { The role of religion } \\
\text { and religiosity in } \\
\text { alcohol consump- } \\
\text { tion in adolescents } \\
\text { in Spain. }\end{array}$ & $\begin{array}{l}\text { Baena BC, Meneses } \\
\text { C, Caperos JM, Prieto } \\
\text { M, Uroz J17. Espa- } \\
\text { nha. 2019. }\end{array}$ & $\begin{array}{l}\text { Estudo descritivo trans- } \\
\text { versal. Amostra: } 2.890 \\
\text { adolescentes. Idade: } 12 \\
\text { a18 anos. }\end{array}$ & $\mathrm{B} ; 2 \mathrm{C}$ & $\begin{array}{l}\text { A religiosidade reduziu o risco do con- } \\
\text { sumo de álcool entre adolescentes, } \\
\text { com diferenças entre as afiliações } \\
\text { religiosas. }\end{array}$ \\
\hline $\begin{array}{l}\text { Spirituality and al- } \\
\text { cohol consumption } \\
\text { among adoles- } \\
\text { cents in Chile: re- } \\
\text { sults of propensity } \\
\text { score stratification } \\
\text { analyses. }\end{array}$ & $\begin{array}{l}\text { Delva J, Han Y, San- } \\
\text { ches N, Andrade FH, } \\
\text { Sanhueza G, Krentz- } \\
\text { manl35. Chile. } 2015 .\end{array}$ & $\begin{array}{l}\text { Estudo descritivo pros- } \\
\text { pectivo. Amostra: } 661 \\
\text { adolescentes. Idade: } 12 \\
\text { a } 19 \text { anos. }\end{array}$ & $\mathrm{B} ; 2 \mathrm{C}$ & $\begin{array}{l}\text { A espiritualidade foi eficaz na prote- } \\
\text { ção ao uso de álcool por adolescen- } \\
\text { tes. }\end{array}$ \\
\hline
\end{tabular}




\begin{tabular}{|c|c|c|c|c|}
\hline $\begin{array}{l}\text { Alcohol use and } \\
\text { church attendance } \\
\text { among seventh } \\
\text { through twelfth } \\
\text { grade students, } \\
\text { Dominican Repu- } \\
\text { blic, } 2011 \text {. }\end{array}$ & $\begin{array}{l}\text { Dohn MN, Méndez } \\
\text { SAJ, Pozo MN, Ca- } \\
\text { brera EA, Dohn AL36. } \\
\text { República Dominica- } \\
\text { na. } 2015 .\end{array}$ & $\begin{array}{l}\text { Estudo descritivo trans- } \\
\text { versal. Amostra: } 3.478 \\
\text { adolescentes. Idade: } 12 \\
\text { a } 18 \text { anos. }\end{array}$ & $B ; 2 C$ & $\begin{array}{l}\text { A religiosidade comportou-se como } \\
\text { fator de proteção aos adolescentes } \\
\text { contra o uso de álcool. }\end{array}$ \\
\hline Fonte: Autoria. & & & & \\
\hline
\end{tabular}

mais antigo foi publicado em 2011 e o mais recente, em 2020. Todos foram disponibilizados na língua inglesa, além de serem classificados com grau de recomendação "B" e nível de evidência " $2 c$ " $(100 \%$; $n=9)$, ou seja, tratava-se de observações de resultados terapêuticos ou estudos descritivos transversais, com evidências insuficientes, contra ou a favor (Quadro 3).

Prevaleceram os publicados na América do Norte $(n=6)$, sendo 5 nos Estados Unidos. Somente 1 foi realizado na América do Sul. Participaram 63.980 adolescentes. Embora se tenha estabelecido como critério de inclusão a faixa etária da adolescência classificada pela Organização Mundial da Saúde, ou seja, dos 10 aos 19 anos, coincidentemente a idade dos participantes compreendeu dos 12 aos 19 anos (Quadro 3).

Tanto a religiosidade quanto a espiritualidade comportaram-se como fatores de proteção a iniciação, frequência e uso do álcool. Evidenciou-se que a religiosidade foi prevalente enquanto protetora, em comparação a espiritualidade, além da influência de fatores sociodemográficos como sexo, raça e afiliação religiosa (Quadro 3).

\section{DISCUSSÃO}

A influência da religiosidade e/ou espiritualidade como fator de proteção ao uso do álcool entre adolescentes despontou como unanimidade entre os estudos desta revisão, reforçando a importância da inserção desta prática na formulação de políticas públicas que visam a promoção e prevenção de agravos à saúde, assim como em seu monitoramento14,17,25,26,32-36. Resumidamente, a proteção referiu-se a atrasar o contato com o álcool, reduzir seu consumo, e, consequentemente, os efeitos deletérios13,36,37.

Embora a maneira como a religiosidade e/ou a espiritualidade se estabeleçam como fatores de proteção não se encontre suficientemente conhecida, frequentar rotineiramente instituições religiosas, possuir uma crença espiritual e maior reprovação do consumo de álcool por pais e amigos comportaram-se como práticas de resguardo ao uso de substâncias psicoativas, incluindo o álcool25,38.

Possivelmente, a proteção se refira ao fato de as redes sociais associadas ao ambiente religioso serem mais receptivas às mensagens anti-álcool do que as seculares36. Em associação, a família e os amigos se configuram como fontes de apoio importantes dentre as que afetam o consumo de álcool, intermediadas pela influência e o convívio7,39-41. De fato, as relações sociais encontram-se fortemente associadas ao uso de álcool por adolescentes, com destaque a influência exercida por amigos que fazem o uso, permeada pelas relações interpessoais estabelecidas por eles8.

Em contrapartida, a aproximação e frequência a templos, incluindo igrejas e sinagogas, a prática de orações individuais e coletivas, meditação, apoio social dos membros da mesma denominação religiosa, possuir identidade religiosa e conectar-se com o transcendente, se configuram como estratégias de fortalecimento e resiliência, traduzidas em hábitos de vida saudáveis que incluem a abstenção do uso do álcool14,26,33,34.

Ainda, evidenciou-se que a religiosidade e/ou a espiritualidade enquanto fator de proteção ao uso do álcool relacionou-se fortemente às variáveis sociodemográficas, como entre adolescentes rurais, brancos e afro-americanos, onde a religiosidade apresentou maior influência de proteção aos brancos apesar de serem mais propensos ao uso do álcool26. Corroborando a esse achado, Vaughan e colaboradores32 identificaram maior impacto entre adolescentes brancos em comparação aos multirraciais, afro-americanos e hispânicos.

O sexo e a denominação religiosa também se apresentaram como variáveis de influência, conforme evidenciado em uma pesquisa norte-americana que incluiu 1.969 adolescentes34. Em outra investigação, identificou-se que as mulheres atrasaram mais o contato com o uso do álcool quando comparadas aos homens14. Pesquisas realizadas no Brasil, México e Hungria, mostraram predomínio do uso do álcool entre adolescentes no sexo masculino8,42,43.

Contudo, na República Dominicana, embora as mulheres tenham apresentado menos episódios de embriaguez e menor consumo médio de álcool em comparação a alunos do sexo masculino, as proporções de uso entre estudantes do sexo masculino e feminino foram iguais, sugerindo mudança das normas sociais conhecidas, que mostravam predomínio do etilismo entre homens. Em resumo, evidenciou-se o risco crescente de problemas relacionados ao álcool entre mulheres jovens 36 .

$\mathrm{Na}$ Hungria, evidenciou-se que os níveis de proteção foram diferentes a depender das afiliações religiosas33, enquanto na Espanha, a ausência ou redução de afiliações religiosas contribuiu para o maior consumo de álcool entre os adolescentes 17. Sabe-se que ter uma religião representava um subconjunto da rede social significativa, onde as experiências, incluindo hábitos de vida 
saudáveis, são compartilhados, além de influenciarem diretamente seus membros 36 .

Em outra investigação realizada nos Estados Unidos, meninas com melhores notas relataram maior desaprovação ao consumo de álcool, enquanto as com maior poder aquisitivo relataram consumir mais32.

De maneira equânime, evidenciaram-se os efeitos protetores, tanto a religiosidade quanto a espiritualidade, como na Hungria, país com histórico de grande consumo de bebidas alcoólicas e pouco espiritualizado33.

Em contrapartida, nos Estados Unidos a espiritualidade apresentou maior efetividade na prevenção e proteção ao uso do álcool em comparação com a religiosidade, enquanto na Espanha, país considerado pouco religioso, evidenciou-se os efeitos protetores da religiosidade 14,17.

$\mathrm{Na}$ contramão da Espanha, embora o Chile se apresenta como um país tradicionalmente religioso, onde $70 \%$ de sua população refira possuir religião e $90 \%$ relate acreditar em Deus, os benefícios referentes ao consumo de álcool entre adolescentes foram evidenciados apenas para aqueles com maiores níveis de espiritualidade, embora os pesquisadores não consideram estes resultados claros e sugeriram a realização de pesquisas longitudinais para confirmar esses achados 35 .

De fato, a religiosidade e espiritualidade, não são sinônimos, e a heterogeneidade entre ambas pode dificultar o pleno entendimento quanto a seus benefícios 44 .

Por fim, considera-se pertinente apontar algumas limitações do presente estudo, que incluem a heterogeneidade metodológica que não permitiu a realização da meta-análise, assim como do predomínio de investigações de delineamento observacional e transversais, portanto, de limitada evidência e grau de recomendação. Assim, investigaçốes controladas e com métodos mais robustos são necessárias, visando compreender melhor e/ou consolidar os achados.

Outros fatores que devem ser pontuados referem-se à subjetividade e amplitude dos aspectos que envolvem a religiosidade e a espiritualidade, que dificultam o estabelecimento de consensos, ao fato de a maioria dos estudos ter incluído apenas adolescentes que frequentavam a escola, excluindo uma população que certamente se configure como mais vulnerável ao uso do álcool, e ao uso de metodologias de coleta de dados fundamentadas exclusivamente no auto relato dos participantes. Assim, os resultados devem ser interpretados com cautela.

No entanto, as contribuições deste estudo à prática clínica são evidentes, ao identificar a religiosidade e/ou a espiritualidade como fator de proteção ao uso de álcool em adolescentes, bem como a maneira como se estabelecem e as variáveis sociodemográficas que a influenciam.

\section{CONCLUSÃO}

Como sugerem os resultados deste estudo, a religiosidade e a espiritualidade configuraram-se como fatores de proteção ao uso do álcool por adolescentes. Nesse sentido, se pesquisas adicionais apoiarem essa hipótese, a identificação de tais adolescentes será importante para destinar prioritariamente os esforços de prevenção. Não obstante, o grande desafio é traduzir esses achados em programas eficazes de prevenção.

\section{REFERÊNCIAS}

1. World Health Organization. Global status report on alcohol and health. Geneva: World Health Organization; 2014.

2. Galduróz JCF, Sanchez ZVM, Opaleye ES, Noto AR, Fonseca AM, Gomes PLS. Factors associated with heavy alcohol use among students in Brazilian capitals. Rev Saude Publica [internet]. 2010;44(2):267-73. Available from: https://doi.org/10.1590/S003489102010000200006

3. Coutinho BLM, Feitosa AA, Diniz CBC, Ramos JLS, Ribeiro LZ, Amorim $\mathrm{SR}$, et al. Alcohol and drugs in adolescence: work process in health in school program. J Human Growth Develop [internet]. 2017;27(1):2834. Available from: https://doi.org/10.7322/jhgd.127646

4. Jorge O, Ferreira RC, Ferreira EP, Vale MP, Kawachi I, Zorzar PM. Binge drinking and associated factors among adolescents in a city in southeastern Brazil: a longitudinal study. Cad Saúde Pública [internet]. 2017;33(2):e00183115. Available from: https://doi. org/10.1590/0102-311x00183115

5. Andrade ME, Santos IHF, Souza AAM, Silva ACS, Leite TS, Oliveira CCC, et al. Experimentation with psychoactive substances by public school students. Rev Saude Publica [internet]. 2017; 51:82. Available from: https://doi.org/10.11606/S1518-8787.2017051006929

6. Raposo JCS, Costa ACQ, Valença PAM, Zarzar PM, Diniz AS, Colares $\checkmark$, et al. Binge drinking and illicit drug use among adolescent students.
Rev Saúde Pública [internet]. 2017;51:83. Available from: https://doi. org/10.11606/s1518-8787.2017051006863

7. Deodato $S$, Nunes $E$, Capelas $M$, Seabra $P$, Sarreira-Santos $A$, Medeiros-Garcia L. Risk behaviors to psychoactive substances use in children and young people in Lisbon. Enferm Glob [internet]. 2017;16(47):98-127. Available from: https://doi.org/10.6018/eglobal.16.3.253011

8. Almeida CS, Abreu MNS, Andrade SN, Abreu MNS, Lana FCF. Factors associated to alcohol use by adolescents. Texto Contexto Enferm [internet]. 2021;30:e20190008. Available from: https://doi. org/10.1590/ 1980-265X-TCE-2019-0008

9. Marshall EJ. Adolescent alcohol use: risks and consequences. Alcohol Alcohol [internet]. 2014;49(2):160-4. Available from: https://doi. org/10.1093/alcalc/agt180

10. Bowden JA, Delfabbro P, Room R, Miller CL, Wilson C. Prevalence, perceptions and predictors of alcohol consumption and abstinence among South Australian school students: a cross-sectional analysis. BMC Public Health [internet]. 2017;17(1):549. Available from: https:// doi.org/10.1186/s12889-017-4475-5

11. Gomes K, Amato TC, Bedendo A, Santos EL, Noto AR. Problems associated with binge drinking among students in Brazil's state capitals. Ciênc Saúde Coletiva [internet]. 2019;24(2):497-507. Available 


\section{REFERÊNCIAS}

\section{from: https://doi.org/10.1590/1413-81232018242.35452016}

12. Silva MP, Fantineli ER, Bacil ED, Piola TS, Malta Neto NA, Campos W. Modificações do consumo de cigarros e bebidas alcoólicas em adolescentes de Curitiba, Paraná: um estudo longitudinal. Ciênc Saúde Coletiva [internet]. 2021;26(6):2365-77. Available from: https://doi. org/10.1590/1413-81232021266.14552019

13. Goncalves IA, Carvalho AAS, Paz EPA. Involvement of adolescents from northern portugal with alcohol. Texto contexto enferm [internet]. 2016;25(4):e4920015. Available from: https://doi.org/10.1590/010407072016004920015

14. Barry AE, Valdez D, Russell AM. Does Religiosity Delay Adolescent Alcohol Initiation? A Long-Term Analysis (2008-2015) of Nationally Representatives Sample of 12th Graders., Subst Use Misuse [internet]. 2020;55(3):503-11. Available from: https://doi.org/10.1080/10 826084.2019.1686024

15. Afifi, RA, Asmar KEL, Bteddini D, Assi M, Yassin N, Bitar S, et al. Bullying Victimization and Use of Substances in High School: Does Religiosity Moderate the Association?. J Relig Health [internet]. 2020;59(1):334-50. Available from: https://doi.org/10.1007/s10943019-00789-8

16. Chen F, Berchtold A, Barrense-Dias Y, Suris JC. Spiritual belief and its link with potentially addictive behaviors in a youth sample in Switzerland. Int J Adolesc Med Health [internet]. 2018;33(1). Available from: https://doi.org/10.1515 / ijamh-2018-0070

17. Baena BC, Meneses C, Caperos JM, Prieto M, Uroz J. The Role of Religion and Religiosity in Alcohol Consumption in Adolescents in Spain. J Relig Health [internet]. 2019;58(5):1477-87. Available from: https://doi.org/10.1007/s10943-018-0694-z

18. Piko BF, Fitzpatrick KM. Substance use, religiosity, and other protective factors among Hungarian adolescents. Add Behav [internet]. 2004;29(6):1095-1107. Available from: https://doi.org/10.1016/j. addbeh.2004.03.022

19. Sanchez ZM, Martins SS, Opaleye ES, Moura YG, Locatelli DP, Noto AR. Social factors associated to binge drinking: a cross-sectional survey among Brazilian students in private high schools. BMC Public Health [internet]. 2011;11:201. Available from: https://doi. org/10.1186/1471-2458-11-201

20. Stevens-Watkins D, Rostosky S. Binge drinking in African American males from adolescence to young adulthood: the protective influence of religiosity, family connectedness, and close friends' substance use. Subst Use Misuse [internet]. 2010;45(10):1435-51. Available from: https://doi.org/10.3109/10826081003754765

21. Stritzke WGK, Butt JC. Motives for not drinking alcohol among Australian adolescents: development and initial validation of a five-factor scale. Addict Behav [internet]. 2001;26(5):633-49. Available from: https://doi.org/10.1016/s0306-4603(00)00147-7

22. Zarzar PM, Jorge KO, Oksanen T, Vale MP, Ferreira EF, Kawachi I. Association between binge drinking, type of friends and gender: a cross-sectional study among Brazilian adolescents. BMC Public Health [internet]. 2012;12:257. Available from: https://doi. org/10.1186/1471-2458-12-257

23. Koenig HG, McCullough M, Larson DB. Handbook of religion and health: a century of research reviewed. New York: Oxford University
Press; 2001.

24. Holmes CJ, Kim-Spoon J. Adolescents Religiousness and Substance Use Are Linked via Afterlife Beliefs and Future Orientation. J Early Adolesc. 2017;37(8):1054-77. Available from: https://doi. org/10.1177/0272431616642325

25. Gryczynski J, Ward BW. Religiosity, Heavy Alcohol Use, and Vicarious Learning Networks Among Adolescents in the United States. Health Educ Behav [internet]. 2012;39(3):341-51. Available from: https://doi.org/10.1177/1090198111417623

26. Dickens DD, Jackman DM, Stanley LR, Swaim RC, Chavez EL. Alcohol consumption among rural African American and White adolescents: The role of religion, parents, and peers. J Ethn Subst Abuse [internet]. 2018;17(3):273-90. Available from: https://doi.org/10.108 $0 / 15332640.2016 .1179155$

27. Higgins J, Green S. Cochrane Handbook for Systematic Reviews of Interventions Version 5.1.0. The Cochrane Collaboration [internet]; 2011. Available from: www.cochrane-handbook.org

28. Moher D, Liberati A, Tetzlaff J, Altman DG. Preferred Reporting Items for Systematic Reviews and Meta-analysis: The PRISMA Statement. PLoS Med [internet]. 2009;6(7):e1000097. Available from: https://doi.org/10.1371/journal.pmed.1000097

29. Moher D, Shamseer L, Clarke M, Ghersi D, Liberati A, Petticrew M, et al. Preferred reporting items for systematic review and meta-analysis protocols (PRISMA-P) 2015 statement. Syst Rev [internet]. 2015;4(1):1. Available from: https://doi.org/10.1186/2046-4053-4-1

30. Beller EM, Glasziou PP, Altman DG, Hopewell S, Bastian $H$, Chalmers I, et al. PRISMA for Abstracts: Reporting Systematic Reviews in Journal and Conference Abstracts. PLoS Med [internet]. 2013;10(4):e1001419. Available from: https://doi.org/10.1371/journal.pmed.1001419

31. Oxford Centre for Evidence-based Medicine. Levels of evidence [internet]. 2009 Available from: http://www.cebm.net/oxford-centre-evidence-based-medicine-levels-evidencemarch-2009

32. Vaughan EL, Dios MA, Steinfeldt JA, Kratz LM. Religiosity, Alcohol Use Attitudes, and Alcohol Use in a National Sample of Adolescents. Psychol Addict Behav [internet]. 2011;25(3):547-53. Available from: https://doi.org/10.1037/a0024660

33. Piko B; Kovacs E, Kriston P; Fitzpatrick K. "To Believe or Not to Believe?" Religiosity, Spirituality, an9d Alcohol Use Among Hungarian Adolescents. J Stud Alcohol Drugs [internet]. 2012;73(4):666-74. Available from: https://doi.org/10.15288/jsad.2012.73.666

34. Hai AH. Are There Gender, Racial, or Religious. Denominational Differences in Religiosity's Effect on Alcohol Use and Binge Drinking Among Youth in the United States? A Propensity Score Weighting Approach. Substance Use Misuse [internet]. 2019;54(7):1096-1105. Available from: https://doi.org/10.1080/10826084.2018.1555598

35. Delva J, Han Y, Sanchez N, Andrade FH, Sanhueza G, Krentzman A. Spirituality and Alcohol Consumption among Adolescents in Chile: Results of Propensity Score Stratification Analyses. Social Work Res [internet]. 2015;39(3):147-57. Available from: https://doi.org/10.1093/ swr/svv019

36. Dohn MN, Jiménez Méndez SA, Pozo MN, Cabrera EA, Dohn A. Al- 


\section{REFERÊNCIAS}

cohol Use and Church Attendance Among Seventh Through Twelfth Grade Students, Dominican Republic, 2011. J Relig Health [internet]. 2014;53(3):675-89. Available from: https://doi.org/10.1007/ s10943-012-9663-0

37. Guimaraes MO, Paiva PCP, Paiva HN, Lamounier JÁ, Ferreira EF, Zarzar PMPA. Religiosity as a possible protective factor against "binge drinking" among 12-year-old students: a population-based study. Ciênc saúde coletiva [internet]. 2018;23(4):1413-123. Available from: http://dx.doi.org/10.1590/1413-81232018234.04872016

38. Scott LD, Hodge DR, White T, Munson MR. Substance use among older youth transitioning from foster care: Examining the protective effects of religious and spiritual capital. Child Fam Soc Work [internet]. 2018;23(3):399-407. Available from: http://dx.doi.org/10.1111/ cfs. 12429

39. Huang GC, Soto D, Fujmoto K, Valente T. The interplay of friendship networks and social networkin sites: longitudinal analysis of selecion and influence effects on adolescente smoking and alcohol use. Am J Public Health [internet]. 2014;104(8):51-59. Available from: https:// doi.org/10.2105/AJPH.2014.302038

40. Whiteman SD, Jensen AC, Maggs J. Similarities and diferences in adolescentes sibling's alchohol - related atitudes, use, adn delinquency: evidence for convergente and divergente influence processes. J
Youth Adolesc [intermet]. 2014;43(5):687-97. Available from: https:// doi.org/10.1007/s10964-013-9971-z

41. Padrão MRAV, Tomasini AJ, Romero MLAM, Silva D, Cavaca AG, Koptcke LS. Peer education: youth protagonism in a preventive approach to alcohol and other drugs. Ciênc Saúde Coletiva [internet]. 2021;26(7):2759-68. Available from: https://doi.org/10.1590/141381232021267.07322021

42. Puente LAR, Castillo BAA, Castillo MMA, Castillo MTA, Garcia NAA, Rodriguez NNO. Consumption of alcohol and tobacco in adolescentes. SMAD, Rev. Eletrônica Saúde Mental Álcool Drog. [internet]. 2016;12(4):200-26. Available from: https://doi.org/10.11606/ issn.1806-6976.v12i4p200

43. Varga S, Piko BF. Being lonely or using substances with friends? A cross- setional study of Hungarian adolescents' health risk behaviours. BMC Public Health [internet]. 2015;12:1107. Available from: https://doi.org/10.1186/s12889-015-2474-y

44. Burris JL, Sauer SE, Carlson CR. A test of religious commitment and spiritual transcendence as independent predictors of underage alcohol use and alcohol-related problems. Psychol Religion Spirit [internet]. 2011;3(3):231-40. Available from: https://doi.org/10.1037/ a0022204 